\title{
Estudo sistemático sobre o potencial de aplicação dos métodos de krigagem em monitoramento de integridade estrutural baseado em impedância eletromecânica
}

\section{Systematic study on the potential application of kriging methods in electromechanical impedance-based structural health monitoring}

Daniel Resende Gonçalves ${ }^{1}$, José dos Reis Vieira de Moura Júnior², Paulo Elias Carneiro Pereira ${ }^{3}$, Henrique Senna Diniz Pinto ${ }^{4}$, Marcos Vinícius Agapito Mendes 5

\section{RESUMO}

A ocorrência de falhas em equipamentos e estruturas civis decorre de diversos fatores, tais como impacto, atrito e fadiga, demandando esforços para sua correção. Entretanto, em virtude de riscos de segurança que tais falhas representam no meio industrial, torna-se necessário o monitoramento de estruturas e equipamentos a fim de identificar rapidamente o início de uma falha, o que permite a tomada de medidas preventivas em tempo hábil. Dados coletados de sensores piezelétricos demandam uma interpolação espacial dos mesmos para a detecção de danos estruturais. Diante deste fato, e considerando que tais informações possuem uma correlação espacial, de tal maneira que são passíveis de serem interpolados espacialmente por métodos de krigagem, foi realizado um estudo sistemático, por meio do Portal de Periódicos da CAPES, acerca da aplicação dos métodos de krigagem em diversas áreas, incluindo a área de mecânica dos sólidos e instrumentação eletromecânica. Foi identificada nesta área uma lacuna de pesquisa que pode ser intensamente explorada, em função da pequena quantidade de publicações (1\% do total) e da vasta aplicabilidade do monitoramento de integridade estrutural baseado em impedância eletromecânica.

Palavras-chave: Krigagem. Monitoramento de Integridade Estrutural. Impedância eletromecânica. Revisão sistemática.
Mestrando em Modelagem e Otimização - Unidade Acadêmica de Matemática e Tecnologia Universidade Federal de Catalão (UFCAT).

E-mail: danielresendeg@gmail.com

${ }^{2}$ Doutor - Unidade Acadêmica de Matemática e Tecnologia Universidade Federal de Catalão (UFCAT).

3,5 Mestre - Unidade Acadêmica de Engenharia - Universidade Federal de Catalão (UFCAT).

${ }^{4}$ Doutor - Unidade Acadêmica de Engenharia - Universidade Federal de Catalão (UFCAT).

The occurrence of failures in equipments and civil structures elapse from several factors, such as impact, friction and fatigue, demanding efforts for its correction. However, due to the security risks that such failures represent in the industrial environment, it's necessary to monitor structures and equipments with the aim of rapidly identify the beginning of a failure, which allows making preventive decisions in a timely manner. Collected data from piezoelectric sensors demands their spatial interpolation for detection of structural damages. Given this fact, and considering the existence of a spatial correlation in these information, being capable to be spatial interpolated by kriging methods, it was performed a systematic literature review, based on CAPES' Portal de Periódicos, about the application of kriging methods in several areas, including the solid mechanics and electromechanial instrumentation one. It was identified a lack of research in this area, which can be intensely explored due to the small amount of papers ( $1 \%$ relative to total) and the wide applicability of the electromechanical impedance-based structural health monitoring.

Keywords: Kriging. Structural Health Monitoring. Electromechanical Impedance. Systematic literature review. 


\section{INTRODUCĀO}

A detecção e correção de falhas em estruturas e equipamentos industriais, geralmente ocasionadas por atrito, impacto e/ou fadiga, são essenciais para o funcionamento adequado do sistema, e envolve a localização, quantificação e estimativa da magnitude da falha (FARRAR; LIEVEN; BEMENT, 2005).

Em função dos elevados custos operacionais e de suporte dos sistemas de engenharia, tornam-se necessários programas de manutenção preditiva baseados no monitoramento do equipamento ou da estrutura (DHILLON, 2008). Através do monitoramento contínuo é possível reduzir custos operacionais através da identificação prévia de uma eventual falha mecânica, antes da ocorrência da mesma, o que permite a execução de ações remediadoras locais e alocação adequada de recursos (YAN; CHEN; MUKHOPADHYAY, 2017; GOPALAKRISHNAN; RUZZENE; HANAGUD, 2011).

O Monitoramento de Integridade Estrutural (Structural Health Monitoring - SHM) dispõe de técnicas para o monitoramento constante/periódico de equipamentos e estruturas, entre as quais, a baseada na impedância eletromecânica (Electro-mechanical Impedance - EMI), cujas vantagens incluem: (1) baixo custo de implantação; e (2) ser uma técnica não invasiva/destrutiva (GIURGIUTIU, 2014; GOPALAKRISHNAN; RUZZENE; HANAGUD, 2011).

A técnica tem sido aplicada para o monitoramento de aeronaves (PAVELKO et al., 2014), equipamentos aeroespaciais (HOSHYARMANESH; ABBASI, 2018; ENCIU; URSU; TOADER, 2017), estruturas de concreto (GUO et al., 2016) e aço (Al et al., 2014), e infraestrutura de sustentação para escavações em solo (ANNAMDAS; YANG, 2012). Além disso, trabalhos tem sido realizados com o propósito de avaliar o efeito da variação da temperatura na coleta das informações dos sensores (transdutores piezelétricos) utilizados para a coleta dos dados (RABELO et al., 2017), e na influência da dimensão dos sensores para o monitoramento (BAPTISTA; VIEIRA FILHO; INMAN, 2011), cujos resultados norteiam o uso da técnica em aplicações industriais.

Dados coletados por meio de sensores piezelétricos fornecem uma informação pontual em termos de métrica de dano (comparação entre as impedâncias atual e inicial da estrutura), de tal maneira que é necessária uma interpolação entre os valores com o propósito de obter a distribuição espacial do dano (localização e intensidade).

Dentre os algoritmos de interpolação, os baseados na geoestatística (krigagem), tem sido utilizados em estudos de modelagem geológica e quantificação de recursos minerais 
(ABEDI; ASGHARI; NOROUZI, 2015; DAYA, 2015; MOOSAVI; GHOLAMNEJAD, 2015; BOISVERT; MANCHUK; DEUTSCH, 2009; RAO; NARAYANA, 2015; BASTANTE et al., 2005), dispersão de poluentes em águas subterrâneas (MACHIWAL et al., 2018; AHAMED; LOGANATHAN, 2017; ELUMALAI et al., 2017; DELBARI; AMIRI; MOTLAGH, 2016; KHANDUZI; PARIZANGANEH; ZAMANI, 2015), mapeamento de qualidade de solos para agricultura (MARTINS et al., 2019; OBROŚLAK; DOROZHYNSKYY, 2017; ZHAOYONG; ABUDUWAILI; YIMIT, 2014), e estudos de variabilidade de chuvas (VENETSANOU et al., 2019; GUPTA; CANLI; LOIGGE; GLADE, 2018; KAMBLE; MACHIWAL, 2017).

Considerando-se a necessidade de interpolação de dados derivados de impedância eletromecânica e a existência de correlação espacial entre resultados pontuais deste parâmetro (GONÇALVES et al., 2020; KRAVOLEC et al., 2018; CHERRIER et al., 2013), condição essencial para a aplicação de métodos de krigagem para interpolação, propõese com este trabalho a realização de uma revisão sistemática da literatura quanto ao desenvolvimento e aplicabilidade dos métodos de Krigagem, incluindo o seu uso como algoritmo interpolador de falhas e suas intensidades, o que pode mostrar o potencial dos métodos de krigagem para monitoramento de estruturas e evidenciar lacunas de pesquisa que podem ser exploradas.

\section{MATERIAIS E METODOS}

A busca das publicações foi realizada no endereço eletrônico do Portal de Periódicos da Coordenação de Aperfeiçoamento de Pessoal de Nível Superior (CAPES) entre os dias 03 de fevereiro de 2020 e 04 de março de 2020. As áreas de aplicação dos métodos de krigagem definidas para a pesquisa foram: (1) geologia e mineração; (2) mapeamento de poluentes/controle ambiental; (3) manejo e conservação do solo/agricultura de precisão; (4) meteorologia, e; (5) mecânica dos sólidos e instrumentação eletromecânica. Para cada área foram definidas palavras-chave na língua inglesa (Tabela 1). 
Tabela 1. Áreas de pesquisa e palavras-chave para busca no Portal Periódicos CAPES

\begin{tabular}{|c|c|c|c|}
\hline Área & Palavra-Chave 1 & Palavra-Chave 2 & Palavra-Chave 3 \\
\hline Geologia e Mineração & kriging & deposit & mining \\
\hline Mapeamento de poluentes/controle ambiental & kriging & pollutant & groundwater \\
\hline $\begin{array}{l}\text { Manejo e conservação do solo/agricultura de } \\
\text { precisão }\end{array}$ & kriging & soil & precision agriculture \\
\hline Meteorologia & kriging & meteorology & rainfall \\
\hline $\begin{array}{c}\text { Mecânica dos sólidos e instrumentação } \\
\text { eletromecânica }\end{array}$ & kriging & $\begin{array}{l}\text { structural health } \\
\text { monitoring }\end{array}$ & $\begin{array}{l}\text { electromechanical } \\
\text { impedance }\end{array}$ \\
\hline
\end{tabular}

A pesquisa foi refinada simultaneamente pelos seguintes parâmetros: período entre 2004 e 2020, e publicações em periódicos com revisão por pares. A partir dos resultados deste refinamento preliminar, os artigos foram filtrados a partir dos seus títulos, e em sequência, pelos resumos. A Tabela 2 mostra o total de publicações para cada busca e critério de refinamento.

Tabela 2. Quantidade de publicações em cada condição de pesquisa e refinamento

\begin{tabular}{cccccc}
\hline & \multicolumn{5}{c}{ Critérios de Refinamento } \\
\cline { 2 - 6 } & $\begin{array}{c}\text { Ausência de } \\
\text { Filtros }\end{array}$ & $\begin{array}{c}\text { Período de } \\
\mathbf{2 0 0 4} \text { à 2020 }\end{array}$ & $\begin{array}{c}\text { Revisão por } \\
\text { pares }\end{array}$ & Título & Resumo \\
\hline $\begin{array}{c}\text { Geologia e Mineração } \\
\begin{array}{c}\text { Mapeamento de poluentes/controle } \\
\text { ambiental }\end{array}\end{array}$ & 1199 & 972 & 964 & 43 & 37 \\
$\begin{array}{c}\text { Manejo e conservação do } \\
\text { solo/agricultura de precisão }\end{array}$ & 760 & 690 & 688 & 37 & 26 \\
$\begin{array}{c}\text { Meteorologia } \\
\text { Mecânica dos sólidos e }\end{array}$ & 1446 & 1311 & 1293 & 80 & 69 \\
instrumentação eletromecânica & 2 & 2 & 2 & 2 & 2 \\
\hline
\end{tabular}

No refinamento baseado nos títulos foram procuradas expressões e contextos relacionados à aplicação dos métodos de krigagem. Na leitura dos resumos foram analisados os seguintes itens: (1) para qual finalidade o método foi usado; (2) se o método apresentou bons resultados diante da pesquisa que estava sendo abordada, e; (3) se o trabalho apresentava uma proposta para o uso do método coerente com o objetivo da busca.

As publicações que atenderam às restrições colocadas foram incluídas no trabalho, a partir das quais foram analisados os seguintes aspectos: (1) quantidade de publicações 
por área; (2) quantidade de publicações dos autores em cada área; (3) quantidade de publicações por periódico, e; (4) quantidade de publicações por ano.

\section{RESULTADOS E DISCUSSÕES}

Os resultados da busca (mapping study) quanto à quantidade de publicações por área (Figura 1) mostraram que duas destas se destacaram: meteorologia $(35,3 \%)$ e manejo e conservação do solo/agricultura de precisão (33,3\%), as quais representaram, conjuntamente, $68,6 \%$ do total de publicações, o que mostra uma grande difusão e aplicabilidade dos métodos de krigagem nestas áreas. As áreas de geologia e mineração, e mapeamento de poluentes/controle ambiental corresponderam a 17,9\% e 12,6\% respectivamente. Já na área de mecânica dos sólidos e instrumentação eletromecânica, a utilização ainda é irrelevante, já que tais publicações representaram apenas 1,0\% do total, de tal maneira há uma lacuna de pesquisa que pode ser eventualmente explorada.

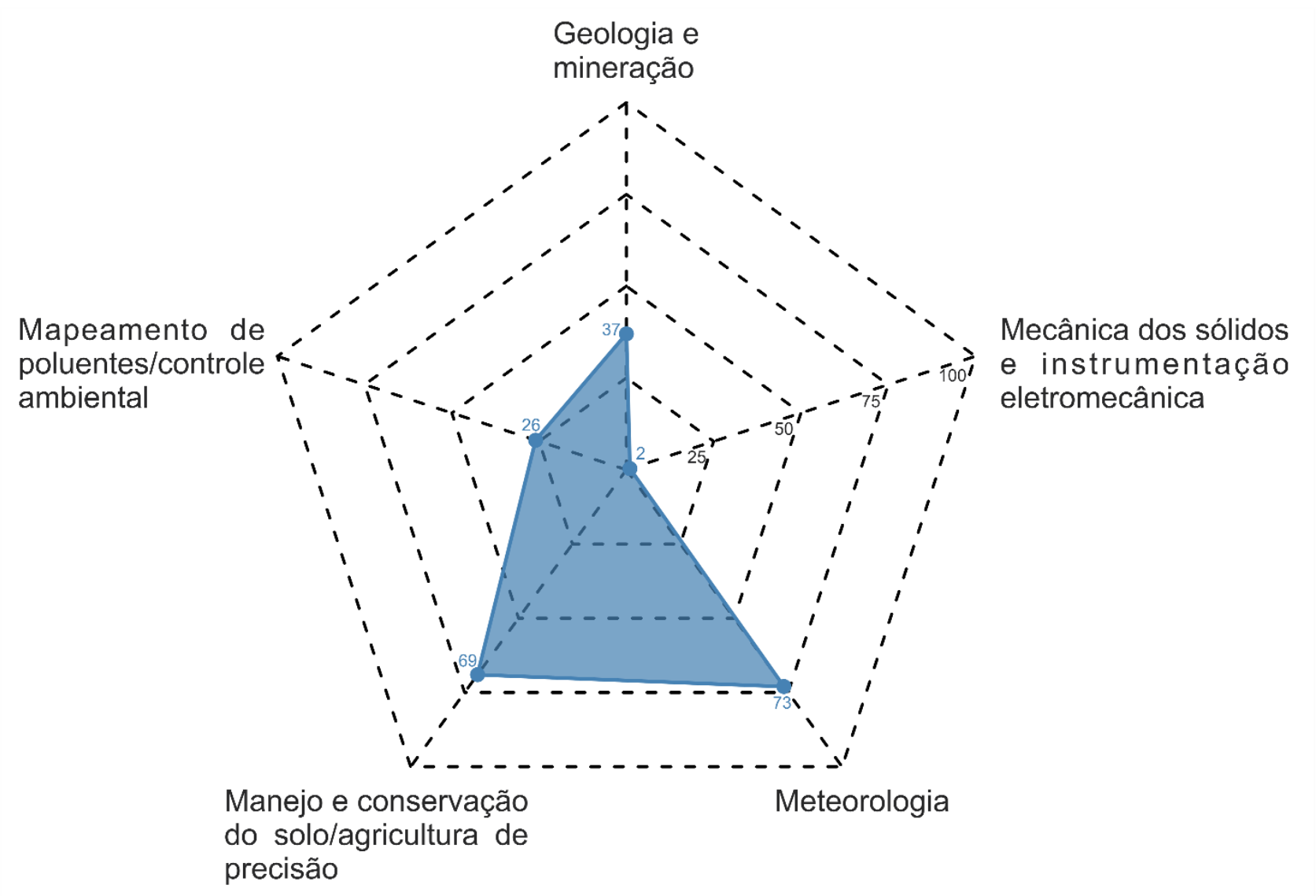

Figura 1. Produções científicas distribuídas por área de busca

Os resultados do mapeamento quanto à quantidade de publicações por autor (Figura 2) mostraram que o grupo com a maior igualdade na distribuição da quantidade de 
publicações por autor foi a "meteorologia", na qual não foram evidenciadas concentrações de publicações em um único autor. Já nas demais tal característica foi observada, particularmente no grupo "manejo e conservação do solo/agricultura de precisão", na qual Piikki, K., representou, sozinho, $25,00 \%$ do total de publicações atribuído aos dez principais autores.

Tais resultados mostram também que em alguns grupos há autores que possuem trabalhos particularmente direcionados para a aplicação dos métodos de krigagem nas áreas em que atuam, enquanto em outros tal característica não ocorre, de tal maneira que nestes as pesquisas relacionadas à aplicação dos métodos de krigagem podem ocorrer ocasionalmente, e de maneira distribuída entre pesquisadores.

No grupo "mecânica dos sólidos e instrumentação eletromecânica", os autores dos trabalhos relativos à aplicação dos métodos de krigagem nesta área possuem, cada um, uma publicação, uma vez que apenas dois trabalhos foram identificados. 


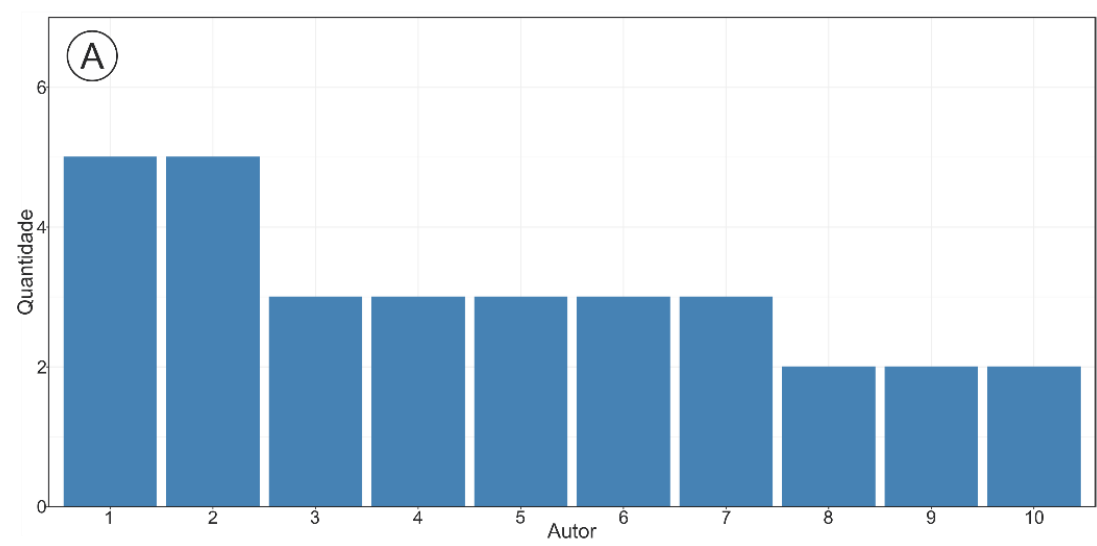

\begin{tabular}{|l|}
\hline \multicolumn{1}{|c|}{ Legenda } \\
1. Castro, J. T. \\
2. Daya, A. A. \\
3. Bastante, F. G. \\
4. González, M. A. S. \\
5. Ordóñez Galán, C. \\
6. Perez, E. G. \\
7. Samanta, B. \\
8. Asghari, O. \\
9. Costa, J. F. C. L. \\
10. Thakur, M. \\
\hline
\end{tabular}

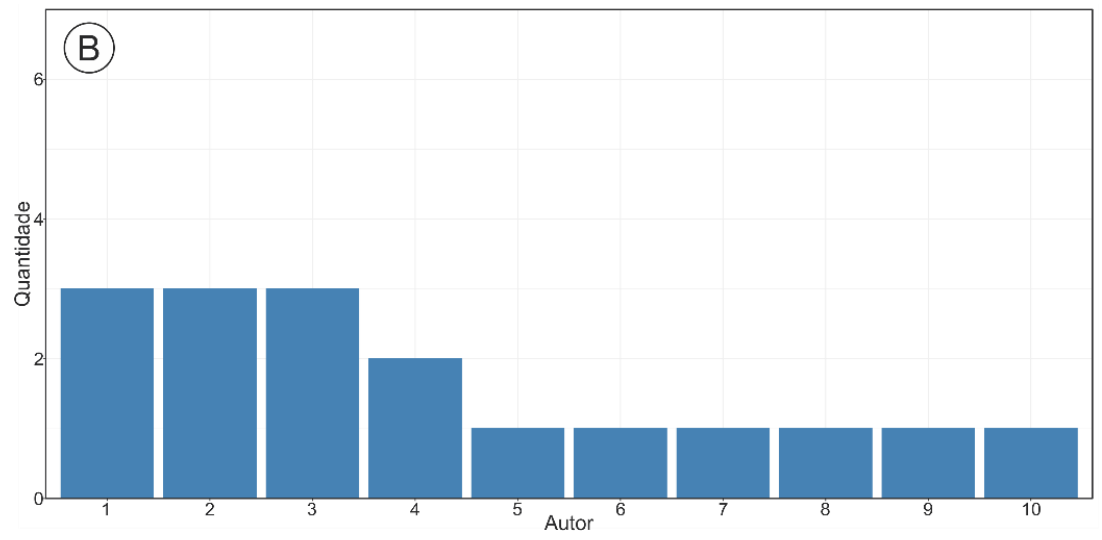

\begin{tabular}{|l|}
\hline \multicolumn{1}{|c|}{ Legenda } \\
1. Adhikary, P. \\
2. Dash, Ch. J. \\
3. Lu, W. \\
4. Chandrasekharan, H. \\
5. Ahamed, A. J. \\
6. Ahmad, M. \\
7. An, Y. \\
8. Khashei-Siuki, A. \\
9. Li, J. \\
10. Teikeu, W. \\
\hline
\end{tabular}

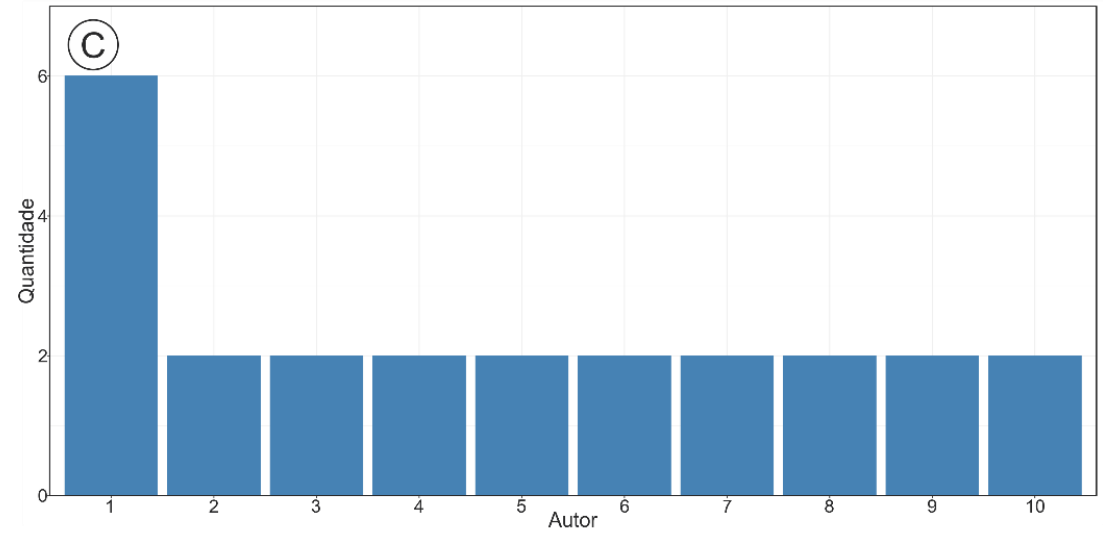

\begin{tabular}{|l|}
\hline \multicolumn{1}{|c|}{ Legenda } \\
1. Piikki, K. \\
2. Casa, R. \\
3. Castrignanò, A. \\
4. Diacono, M. \\
5. Fortes, R. \\
6. Ge, Y. \\
7. Gholizadeh, A. \\
8. Kerry, R. \\
9. Moral, F. J. \\
10. Wu, Yi-Hwa \\
\hline
\end{tabular}

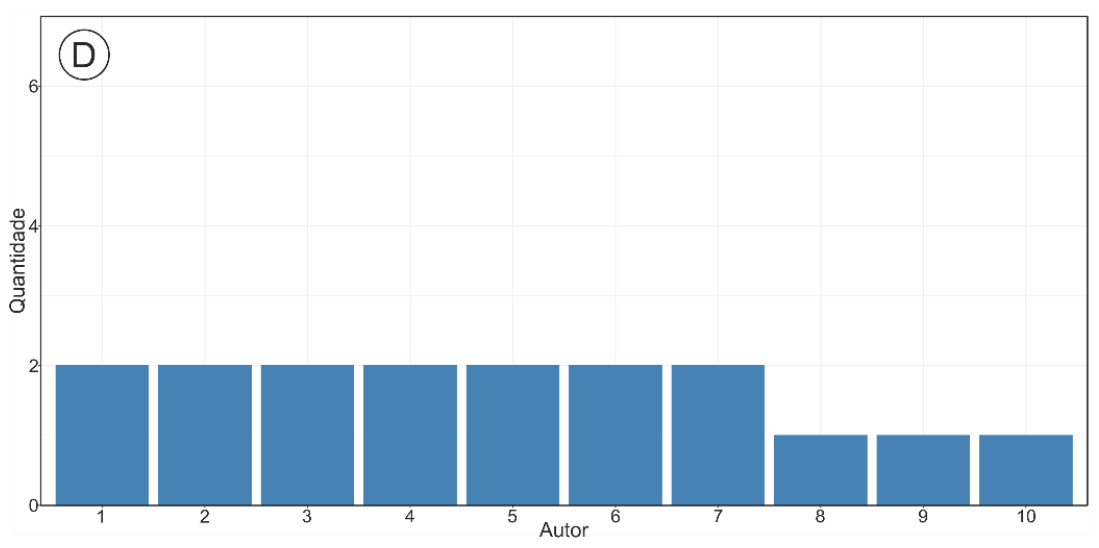

\begin{tabular}{l} 
Legenda \\
1. Ayanlade, A. \\
2. Kisaka, M. O. \\
3. Mairura, F. \\
4. Mucheru-Muna, M. \\
5. Mugendi, D. \\
6. Mugwe, J. N. \\
7. Park, No-Wook \\
8. Kizza, M. \\
9. Sivapragasam, C. \\
10. Teng, H. \\
\hline
\end{tabular}

Figura 2. Inventário da quantidade de publicações por autor nas áreas de geologia e mineração (A); mapeamento de poluentes/controle ambiental; (B); manejo e conservação do solo/agricultura de precisão (C); e meteorologia (D). 
O mapeamento da quantidade relativa (proporção) de publicações acerca dos métodos de krigagem por periódico (Figura 3) mostrou que no grupo "manejo e conservação do solo/agricultura de precisão" o periódico com a maior quantidade de publicações do tema (Precision Agriculture) representou, sozinho, $24,6 \%$ do total de publicações do grupo, o que o caracteriza como uma referência no tema (krigagem) em relação aos demais da mesma área. Já nos demais grupos, as quantidades relativas atribuídas aos respectivos periódicos de maior relevância no tema são menores: 19,2\%, 13,5\% e 12,3\% atribuídos aos periódicos "Environmental Monitoring and Assessment" (mapeamento de poluentes/controle ambiental), "Mathematical Geosciences" (geologia e mineração), e "Theoretical and Applied Climatology" (meteorologia).
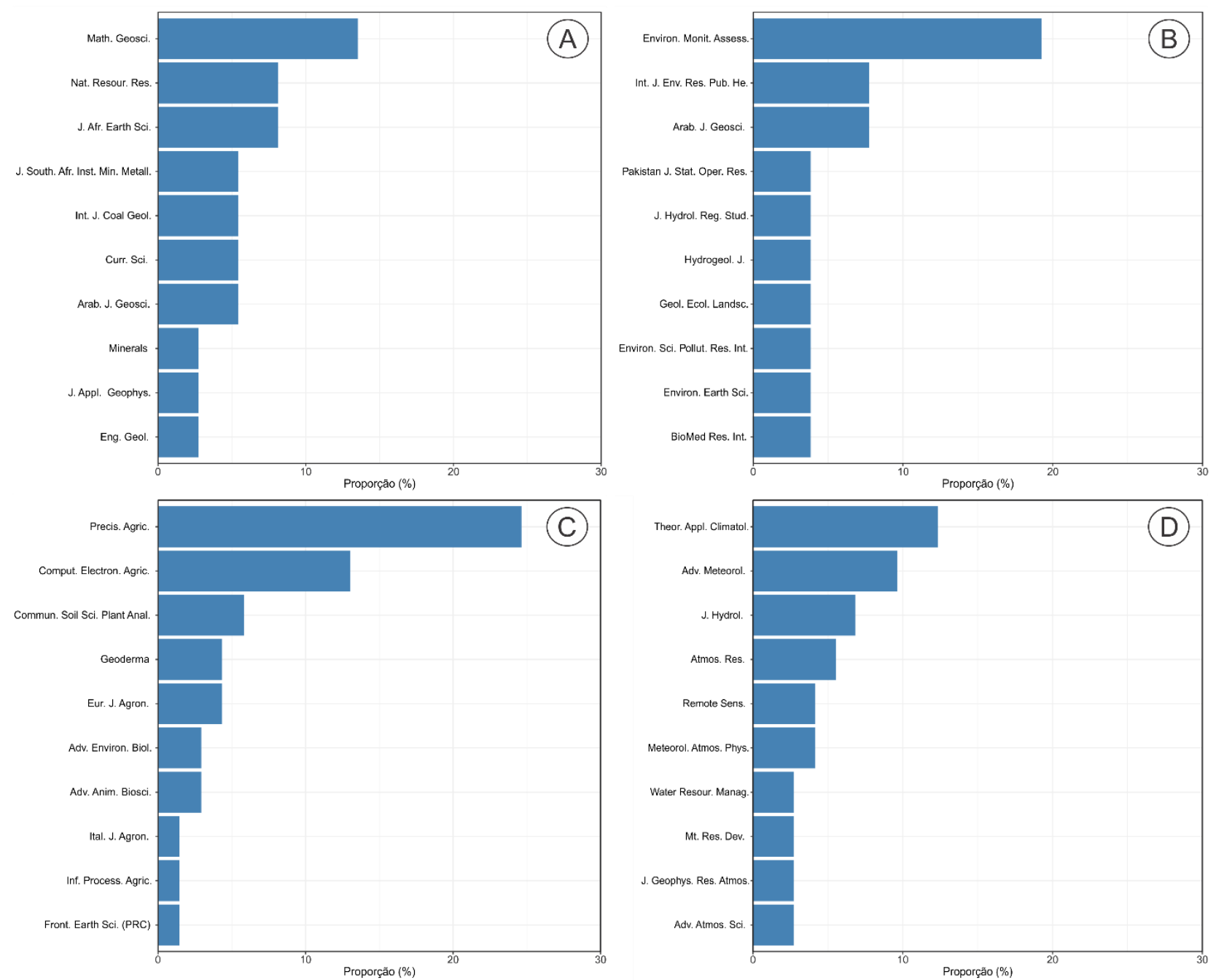

Figura 3. Inventário da proporção de publicações por periódico nas áreas de geologia e mineração $(A)$; mapeamento de poluentes/controle ambiental; $(B)$; manejo e conservação do solo/agricultura de precisão (C); e meteorologia (D). 
Na Figura 3 também é possível observar que no grupo "meteorologia" as publicações associadas ao tema (krigagem) ocorrem mais distribuídas nos periódicos, de tal maneira que não há um periódico aparentemente direcionado para as aplicações de krigagem na solução de problemas específicos da área, contrariamente ao que ocorre no grupo "manejo e conservação do solo/agricultura de precisão", em que um único periódico foi responsável por $24,6 \%$ do total de publicações do grupo.

No que tange ao grupo "mecânica dos sólidos e instrumentação eletromecânica", foram identificados apenas dois periódicos com publicações sobre a utilização dos métodos de krigagem para o monitoramento de integridade estrutural: HOLOS (GONÇALVES et al., 2020) e Journal of Nondestructive Evaluation (MATSUZAKI; KAWASAKI; TODOROKI, 2015).

Os resultados da quantificação do total de publicações por ano (Figura 4) mostraram que entre 2004 e 2015, houve, de maneira geral, um aumento desse valor, com uma taxa de crescimento anual média de $81 \%$, cujo maior volume de publicações ocorreu em 2015 , após o qual houve uma diminuição progressiva da quantidade de publicações no tema, com uma taxa de decrescimento anual média de 33\%, o que pode ser uma consequência da utilização de outra metodologia na solução de problemas pertinentes à cada área.

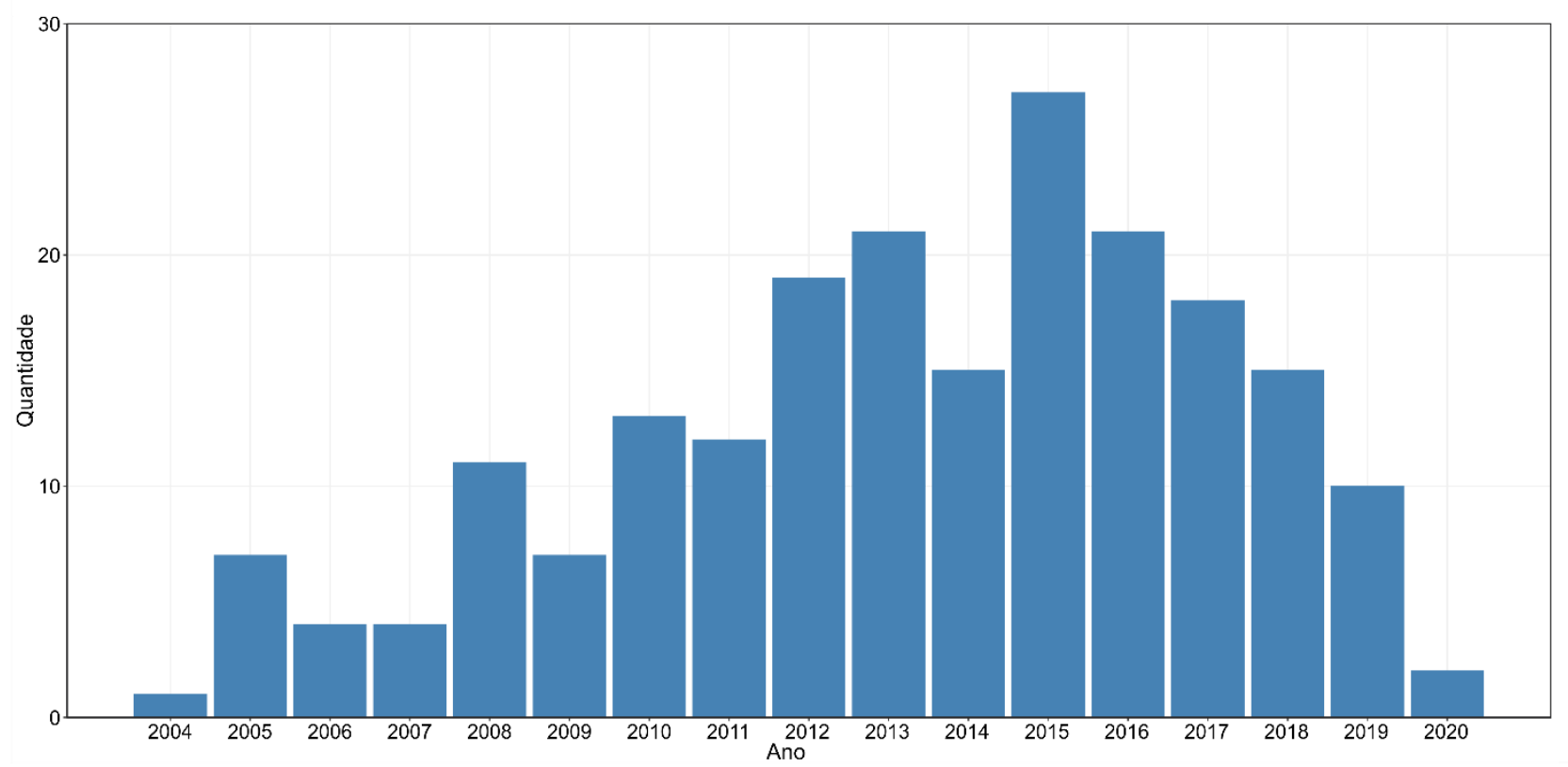

Figura 4. Quantidade total de publicações por ano. 


\section{CONSIDERACÓES FINAIS}

A partir da pesquisa sistemática realizada foi possível mapear: (1) quantidade de publicações por grupo de busca; (2) publicações por autor; (3) publicações por periódico, e; (4) quantidade de publicações por ano.

Os resultados mostraram que os métodos de krigagem são utilizados principalmente nas áreas de meteorologia e de manejo e conservação do solo/agricultura de precisão, as quais responderam por 35,3\% e 33,3\%, respectivamente, em relação ao total de artigos publicados no período analisado. Em relação às demais áreas, há pouquíssimas pesquisas relacionadas à aplicação dos métodos de krigagem, em especial na área de mecânica dos sólidos e instrumentação eletromecânica, a qual respondeu por apenas $1,0 \%$ do total.

Tais resultados mostram que há uma lacuna de pesquisa que pode ser eventualmente explorada na área de mecânica dos sólidos e instrumentação eletromecânica, principalmente diante do fato de que dados coletados de sensores piezelétricos possuem uma correlação espacial, condição necessária para a aplicação de métodos de krigagem para interpolação, com a finalidade de detectar danos estruturais em equipamentos e/ou estruturas.

\section{REFERENCIAS}

ABEDI, M.; ASGHARI, O.; NOROUZI, G. H. Collocated cokriging of iron deposit based on a model of magnetic susceptibility: a case study in Morvarid mine, Iran. Arabian Journal of Geosciences. Heidelberg, v. 8, abr. 2015, p. 2179-2189.

AHAMED, A. J.; LOGANATHAN, K. Water quality concern in the Amaravathi River Basin of Karur district: a view at heavy metal concentration and their interrelationships using geostatistical and multivariate analysis. Geology, Ecology, and Landscapes. Londres, Inglaterra, v. 1, n. 1, 20 mar. 2017, p. 19-36.

Al, D. et al. An effective electromechanical impedance technique for steel structural health monitoring. Construction and Building Materials. Oxfordshire, Inglaterra, v. 73, 16 out. 2014, p. 97-104.

ANNAMDAS, V. G. M.; YANG, Y. Practical implementation of piezo-impedance sensors in monitoring of excavation support structures. Structural Control \& Health Monitoring. Chichester, Inglaterra, v. 19, n. 2, mar. 2012, p. 231-245.

BAPTISTA, F. G.; VIEIRA FILHO, J.; INMAN, D. J. Sizing PZT Transducers in ImpedanceBased Structural Health Monitoring. IEEE Sensors Journal. Piscataway, Estados Unidos da América, v. 11, n. 6, jun. 2011, p. 1405-1414. 
BASTANTE, F. G. et al. Evaluation of the resources of a slate deposit using indicator kriging. Engineering Geology. Amsterdam, v. 81, 24 out. 2005, p. 407-418.

BOISVERT, J. B.; MANCHUK, J. G.; DEUTSCH, C. V. Kriging in the Presence of Locally Varying Anisotropy Using Non-Euclidean Distances. Mathematical Geosciences. Heidelberg, v. 41, 10 jun. 2009, p. 585-601.

CANLI, E.; LOIGGE, B.; GLADE, T. Spatially distributed rainfall information and its potential for regional landslide early warning systems. Natural Hazards. Cham, Suíça, v. 91, n. 1, abr. 2018, p. 103-127.

CHERRIER, P. S. O. et al. Damage localization map using electromechanical impedance spectrums and inverse distance weighting interpolation: Experimental validation on thin composite structures. Structural Health Monitoring. Londres, Inglaterra, v. 12, n. 4, 01 jul. 2013, p. 311-324.

DAYA, A. A. Application of median indicator kriging in the analysis of an iron mineralization. Arabian Journal of Geosciences. Heidelberg, v. 8, jan. 2015, p. 367-377.

DELBARI, M.; AMIRI, M.; MOTLAGH, M. B. Assessing groundwater quality for irrigation using indicator kriging method. Applied Water Science. Cham, Suíça, v. 6, nov. 2016, p. 371-381.

DHILLON, B. S. Mining Equipment Reliability, Maintainability, and Safety. 1. ed. Londres, Inglaterra: Springer-Verlag London, 2008.

ELUMALAI, V. et al. Spatial interpolation methods and geostatistics for mapping groundwater contamination in a coastal area. Environmental Science and Pollution Research. Heidelberg, Alemanha, v. 24, abr. 2017, p. 11601-11617.

ENCIU, D.; URSU, I.; TOADER, A. New results concerning structural health monitoring technology qualification for transfer to space vehicles. Structural Control \& Health Monitoring. Chichester, Inglaterra, v. 24, n. 10, 06 set. 2017, p. 1-14.

FARRAR, C. R.; LIEVEN, N. A.; BEMENT, M. An Introduction to Prognosis. In: INMAN, D. J. et al. (Eds.). Damage Prognosis: For Aerospace, Civil and Mechanical Systems. 1. ed. Chichester, Inglaterra: John Wiley \& Sons Ltd, 2005. p. 1-12.

GONÇALVES, D. R.; MOURA JÚNIOR, J. R. V.; PEREIRA, P. E. C. Monitoramento de integridade estrutural baseado em impedância eletromecânica utilizando o método de krigagem ordinária. HOLOS. Natal, Brasil, v. 36, n. 2, abr. 2020, p. 1-17.

GOPALAKRISHNAN, S.; RUZZENE, M.; HANAGUD, S. Computational Techniques for Structural Health Monitoring. 1. ed. Londres, Inglaterra: Springer-Verlag London, 2011.

GUO, F. et al. Practical Issues Related to the Application of Electromechanical Impedance-Based Method in Concrete Structural Health Monitoring. Research in 
Nondestructive Evaluation. Filadélfia, Estados Unidos da América, v. 27, n. 1, 01 mar. 2016, p. 26-33.

GUPTA, A.; KAMBLE, T.; MACHIWAL, D. Comparison of ordinary and Bayesian kriging techniques in depicting rainfall variability in arid and semi-arid regions of north-west India. Environmental Earth Sciences. Nova York, Estados Unidos da América, v. 76, n. 15, ago. 2017, p. 1-16.

HOSHYARMANESH, H.; ABBASI, A. Structural health monitoring of rotary aerospace structures based on electromechanical impedance of integrated piezoelectric transducers. Journal of Intelligent Material Systems and Structures. Londres, Inglaterra, v. 29, n. 9, 12 fev. 2018, p. 1799-1817.

KHANDUZI, F.; PARIZANGANEH, A.; ZAMANI, A. Application of multivariate statistics and geostatistical techniques to identify the spatial variability of heavy metals in groundwater resources. Caspian Journal of Environmental Sciences. Rasht, Irã, v. 13, n. 4, 2015, p. 333-347.

KRALOVEC, C.; SCHAGERL, M.; MAYR, M. Localization of damages by model-based evaluation of electro-mechanical impedance measurements. The e-Journal of Nondestructive Testing. Bad Breisig, Alemanha, v. 23, n. 11, 2018, p. 1-12.

MACHIWAL, D. et al. A review of GIS-integrated statistical techniques for groundwater quality evaluation and protection. Environmental Earth Sciences. Nova York, Estados Unidos da América, v. 77, n. 19, out. 2018, p. 1-30.

MARTINS, R. N. et al. Accuracy Assessments of Stochastic and Deterministic Interpolation Methods in Estimating Soil Attributes Spatial Variability. Communications in Soil Science and Plant Analysis. Londres, Inglaterra, v. 50, n. 20, 26 set. 2019, p. 25702578.

MOOSAVI, E.; GHOLAMNEJAD, J. Long-Term Production Scheduling Modeling for the Open Pit Mines Considering Tonnage Uncertainty via Indicator Kriging. Journal of Mining Science. Rússia, v. 51, n. 6, 2015, p. 1226-1234.

OBROŚLAK, R.; DOROZHYNSKYY, O. Selection of a semivariogram model in the study of spatial distribution of soil moisture. Journal of Water and Land Development. Raszyn, Polônia, v. 35, n. 1, 19 dez. 2017, p. 161-166.

PAVELKO, I. et al. Bolt-joint structural health monitoring by the method of electromechanical impedance. Aircraft Engineering and Aerospace Technology. Bradford, Inglaterra, v. 86, n. 3, 29 abr. 2014, p. 207-214.

RABELO, D. S. et al. Impedance-based structural health monitoring and statistical method for threshold-level determination applied to 2024-T3 aluminum panels under varying temperature. Structural Health Monitoring. Londres, Inglaterra, v. 16, n. 4, 01 jul. 2017, p. 365-381. 
RAO, V. K.; NARAYANA, A. C. Application of nonlinear geostatistical indicator kriging in lithological categorization of an iron ore deposit. Current Science. Índia, v. 108, n. 3, 10 fev. 2015, p. 413-421.

VENETSANOU, P. et al. Minimizing the uncertainties of RCMs climate data by using spatio-temporal geostatistical modeling. Earth Science Informatics. Cham, Suíça, v. 12, n. 2, 01 jun. 2019, p. 183-196.

YAN, R.; CHEN, X.; MUKHOPADHYAY, S. C. Advanced Signal Processing for Structural Health Monitoring. In: YAN, R. et al. (Eds.). Structural Health Monitoring: An Advanced Signal Processing Perspective. 1. ed. Cham, Suíça: Springer International AG, 2017. p. 111.

ZHAOYONG, Z.; ABUDUWAILI, J.; YIMIT, H. The Occurrence, Sources and Spatial Characteristics of Soil Salt and Assessment of Soil Salinization Risk in Yanqi Basin, Northwest China. PLoS One. São Francisco, Estados Unidos da América, v. 9, n. 9, 11 set. 2014, p. 1-12. 